\title{
Pathogenic Spectrum and Resistance Pattern of Bloodstream Infections Isolated from Postpartum Women: A Multicenter Retrospective Study
}

\author{
Qin Zou' \\ Hua Zou' \\ Yan Shen' \\ Lang Yu' \\ Wei Zhou (D) ${ }^{2}$ \\ Chenglin Sheng ${ }^{3}$ \\ Ang Liao ${ }^{4}$ \\ Chunli Li' \\ 'Department of Laboratory Medicine, \\ Chongqing Health Center for Women \\ and Children, Chongqing, People's \\ Republic of China; ${ }^{2}$ Department of \\ Obstetrics and Gynecology, Chongqing \\ Health Center for Women and Children, \\ Chongqing, People's Republic of China; \\ ${ }^{3}$ Department of Laboratory Medicine, \\ Wanzhou Health Center for Women and \\ Children, Chongqing, People's Republic of \\ China; ${ }^{4}$ Department of Laboratory \\ Medicine, Yongchuan Health Center for \\ Women and Children, Chongqing, \\ People's Republic of China
}

Purpose: Bloodstream infections (BSIs) cause morbidity and mortality in postpartum patients, resulting in poor prognosis for both mother and neonate. Gram-negative bacteremia is a public health threat, with high mortality among vulnerable populations and significant global economic costs. Gram-negative bacteremia and antimicrobial resistance are increasing. This study retrospectively analyzed the pathogen distribution and drug sensitivity among postpartum patients with BSIs to identify appropriate antibacterial agents for perioperative therapy.

Material and Methods: All bacteremia cases between January 2015 and December 2020 from three Health Centers for Women and Children in Chongqing, China, were retrospectively reviewed. Clinical data were collected from medical records and charts. Blood samples were cultured by BD BACTEC FX200. Bacterial and fungal species and bacterial susceptibility were identified by a BD Phoenix ${ }^{\mathrm{TM}}$ M50 automatic detection machine.

Results: In total, 274 pathogenic strains were isolated from 272 blood samples. Excluding 25 suspected contamination strains, 248 blood samples yielded 249 microorganisms, including 214 gram-negative bacteria (85.9\%), 34 gram-positive bacteria (13.6\%), and 1 fungus $(0.5 \%)$. Escherichia coli (E. coli) was the most frequently isolated pathogen, both overall and among gram-negative bacilli (73.5\%). Streptococcus agalactiae represented $3.6 \%$ of grampositive cocci $(n=9)$. Laboratory-confirmed anaerobic infections comprised $9.2 \%$ of cases $(n$ $=23$ ). Additionally, $47.4 \%$ of postpartum patients with BSIs suffered premature rupture of membranes (PROM), a suspected infection risk factor. Drug sensitivity levels remained unchanged for less commonly used drugs, but resistance increased against commonly used drugs. Specifically, E. coli resistance against fourth-generation cephalosporins increased during this study period.

Conclusion: $E$. coli is the most common gram-negative bacillus in postpartum patients with BSIs, and increased anaerobic bacterial detections suggest genital tract inflammation control before delivery is necessary. Effective drug resistance monitoring remains necessary to alleviate bacterial resistance, such as preventing inappropriate antibiotic applications.

Keywords: bacteremia, Escherichia coli, anaerobic bacterial, perioperative therapy

\section{Introduction}

Despite national and international efforts to improve maternal outcomes, infectionrelated maternal morbidity and mortality rates remain a large health care concern. Postpartum infections, a subset of maternal infections that occur between delivery and the 42 nd day postpartum ${ }^{1}$ represent the fifth most common cause of maternal death, behind only hypertensive disorders, obstetric hemorrhage, abortion/ectopic
Department of Laboratory Medicine, Chongqing Health Center for Women and Children, Chongqing, 40II47,

People's Republic of China

Tel +86 23-603544559

$\mathrm{Fax}+8623-63840868$

Email IcI5I8023@I26.com 
gestations, and obstetric embolism. ${ }^{2,3}$ Improved understanding of postpartum infection is a key to achieving sustainable development goals (SDGs) and executing strategies targeting the reduction of preventable maternal and neonatal mortality.

The most common postpartum infections include endometritis, urinary tract infections, surgical site infections, bloodstream infections (BSI), and wound infections. ${ }^{1,4}$ In particular, BSIs represent common and serious clinical infections and are among the top seven causes of overall mortality in Europe and the 11th leading cause of death in the USA. ${ }^{5}$ The fatality rate of BSIs, based on the general population of mainland China, is approximately $20 \%{ }^{6}$ The early diagnosis and rational use of effective antibacterial drugs are key steps to treating patients with BSI. The delayed or inappropriate use of antibacterial drugs can lead to deterioration and complications. ${ }^{5}$ Additionally, the pathogenic spectrum and pattern of antimicrobial resistance associated with BSIs often differ across affected regions due to differences in epidemiological and geographic features and the clinical use of antibacterial drugs in different regions over time. ${ }^{7,8}$ Understanding the prevalence of different types of microorganisms and their antimicrobial resistance characteristics will better guide physicians, improve infection control, and inform policymakers in various countries and regions when making evidence-based decisions to overcome antimicrobial resistance. $^{9,10}$

This multicenter, retrospective study was performed to determine the distribution of pathogens and drug sensitivities in puerperal microbiologically identified BSI, focusing on the antimicrobial resistance of Escherichia coli. This information can guide antimicrobial stewardship programs and infection control activities in hospitals.

\section{Materials and Methods}

\section{Data Collection}

By reviewing the medical records of postpartum patients, we retrospectively collected data from the information systems of three hospitals and laboratories between January 2015 and December 2020. The basic information gathered included gestational age, obstetric history, laboratory values, delivery information, and maternal age, as shown in Table 1. Patients with acute pulmonary embolism, amniotic fluid embolism, adverse drug reactions, drug fever, viral infection, autoimmune conditions, and transfusion reactions were excluded from this study. We
Table I Demographic Details for Postpartum Women from 2015 to 2020

\begin{tabular}{|l|l|}
\hline Patient Characteristic & No. of Cases \\
\hline $\begin{array}{l}\text { Age (years) } \\
<35\end{array}$ & $29.3 \pm 3.7$ \\
$\geq 35$ & $223(89.9 \%)$ \\
\hline $\begin{array}{l}\text { Gestational age (weeks) } \\
<37\end{array}$ & $37.5 \pm 4.2$ \\
$\geq 37$ & $60(24.2 \%)$ \\
\hline Delivery mode & $188(75.8 \%)$ \\
Vaginal delivery & \\
Cesarean section & $26(10.5 \%)$ \\
Abortion & $220(88.7 \%)$ \\
\hline Fever onset (day) & $2(0.8 \%)$ \\
\hline Temperature & $2.1 \pm 1.4$ \\
\hline PROM & $38.6 \pm 0.7$ \\
\hline Laboratory characteristics & $118(47.4 \%)$ \\
WBC & \\
CRP & $12.9(10.8-15.8)$ \\
\hline PCT & $91.8(65.4-150)$ \\
\hline Pathogens & $0.34(0.18-0.75)$ \\
Gram-negative bacteria & \\
Gram-positive bacteria & $214(85.9 \%)$ \\
\hline Fungus & $34(13.6 \%)$ \\
\hline
\end{tabular}

Abbreviations: PROM, premature rupture of membranes; WBC, white blood cell; CRP, C-reactive protein; PCT, Procalcitonin.

reviewed the electronic medical records to determine whether the patient received any antimicrobial agents during the 15 days prior to delivery or underwent any recent surgeries before the onset of bacteremia. The primary focus of infection was determined based on the clinical presentation and a final diagnosis made by the primary care clinician.

\section{Ethics Statement}

This study was performed as a multicenter, non-interventional, retrospective study conducted at three Health Centers for Women and Children, in accordance with all relevant regulations in Chongqing, China. The study protocol was reviewed and approved by the Institutional Review Board/Independent Ethics Committee at each study site, including Chongqing Health Center for Women and Children, Wanzhou Health Center for Women and Children, and Yongchuan Health Center for Women and Children. Although informed consent was not obtained, patients were given the opportunity to decline 
permission for their clinical records to be used for research (opt-out consent provision). Patient privacy and confidentiality of data were maintained in accordance with the Declaration of Helsinki.

\section{Microorganism Identification and Antimicrobial Susceptibility Testing}

Two aerobic and two anaerobic blood samples ( $5 \mathrm{~mL}$ each) were collected aseptically from two different peripheral veins and incubated in the BD BACTECTM FX200 (BD Company, USA) automated blood culture system for 7 days before reporting no growth. The detected positive strains were inoculated onto blood agar plates (BAPs) and chocolate agar plates (Pangtong Medical, Chongqing, China) and incubated aerobically for 18-24 hours in a humid chamber (Thermo Fisher Scientific, USA) containing $5 \% \mathrm{CO}_{2}$ at $37{ }^{\circ} \mathrm{C}$ for subculture. Pure colonies of isolated bacteria were emulsified in $2 \mathrm{~mL} 0.85 \%$ normal saline. Bacterial suspensions with an optical density of $0.5-0.6$ at $600 \mathrm{~nm}$ wavelength were considered to have acceptable bacterial concentrations. Reagents were then immersed to the suspension and transferred to the $\mathrm{BD}$ PhoenixTM M50 (BD Company) identification system for bacterial identification and susceptibility testing.

Antibiotic breakpoints were defined using the European Committee on Antimicrobial Susceptibility Testing (EUCAST) guidelines version 5.0 and Clinical and Laboratory Standards Institute (CLSI) M100-S27th. ${ }^{11,12}$

\section{Data Quality Control}

All data collection and recording steps were monitored. The reagents were checked for expiration dates and maintained under appropriate storage conditions, including acceptable temperature and humidity. Standard operating procedures (SOPs) were prepared and strictly followed. The quality of the culture media and antimicrobial susceptibility testing were verified by using standard quality control strains: E. coli ATCC 25922, Staphylococcus aureus ATCC 29213, Enterococcus faecalis ATCC29212, and Klebsiella pneumoniae ATCC700603. The inoculum densities of the bacterial suspensions were standardized using $0.5 \%$ McFarland standards for susceptibility testing.

\section{Definition}

Patients who developed fever $>38.0^{\circ} \mathrm{C}$ or hypothermia $<36.0^{\circ} \mathrm{C}$ underwent blood cultures, procalcitonin, and $\mathrm{C}$-reactive protein testing. In the case of potential common skin commensals, such as coagulase-negative staphylococci, Propionibacterium acnes, Clostridium, Corynebacterium diphtheriae, Acinetobacter bacilli, or other common bacteria in the environment, BSI was defined as the laboratory-confirmed growth of a potential pathogen in at least two consecutive positive blood cultures drawn on two separate occasions, to eliminate the possibility of contamination during the collection or cultivation of blood samples. ${ }^{13}$

\section{Data Analysis}

All analyses were performed using SPSS ${ }^{\mathrm{TM}}$ software, version 21.0 (IBM Corporation, Armonk, NY, USA). The frequencies and proportions of isolates resistant to the tested antibiotics and other categorical data were calculated. Continuous variables were analyzed by Student's $t$-test or by the Mann-Whitney $U$-test, depending on the sample distribution. Normally distributed data are described as the mean \pm standard deviation $(\mathrm{M} \pm \mathrm{SD})$, whereas non-normally distributed data are described as the median and range.

\section{Results \\ Patient Characteristics}

For the study period from January 2015 to December 2020, a total of 272 non-duplicate BSI-positive samples from postpartum patients were enrolled, and the patient characteristics are summarized in Table 1 . In the overall cohort, all participants met the criteria for one or more postpartum infections. 274 pathogenic strains were isolated from 272 blood samples. Excluding 25 suspected contamination strains, laboratory-confirmed BSI was diagnosed in 248 BSI samples, yielding 249 organisms. The isolated organisms were predominantly gram-negative bacilli, regardless of maternal age, gestational week, or delivery mode. According to the data, 220 participants (88.7\%) delivered through cesarean section, and 106 cases $(48.2 \%)$ suffered from premature rupture of membranes (PROM), with E. coli being the most commonly isolated pathogen. Additionally, the onset of fever among participants typically occurred within two days after delivery according to Table 1 .

\section{E. coli Dominates BSI in Postpartum Patients}

Of a total 249 isolated microorganisms, excluding coagulase-negative Staphylococcus (CoNS) strains that were suspected of representing contamination, $85.9 \%$ of isolates 
$(\mathrm{n}=214)$ were identified as gram-negative bacteria, $13.6 \%$ of isolated strains were gram-positive bacteria $(\mathrm{n}=34)$, and $0.4 \%$ of strains were identified as fungus $(\mathrm{n}=1$; Table 1). E. coli $(\mathrm{n}=183)$ was the most frequently identified gram-negative BSI-causative pathogen in postpartum patients, accounting for $85.5 \%$ of gram-negative strains, as presented in Figure 1A. Klebsiella pneumoniae $(\mathrm{n}=11)$ contributed to $5.0 \%$ of patients with BSI. Morganell amorganii, Burkholderia cepacia, Citrobacter kocher, and Citrobacter farmer each occurred in $0.5 \%$ of BSI-positive samples, with one sample that remained unidentified. Additionally, $7.0 \%$ of gram-negative strains isolation from blood culture-positive samples were anaerobes $(\mathrm{n}=$ 15). As shown in Figure 1B, approximately $13.6 \%$ of isolated strains were gram-positive bacteria $(\mathrm{n}=34)$, with the majority belonging to Streptococcus $(\mathrm{n}=18)$, accounting for $52.9 \%$, followed by Enterococcus faecalis $(\mathrm{n}=6,17.6 \%)$, anaerobes $(\mathrm{n}=8,23.5 \%)$, and Staphylococcus aureus $(\mathrm{n}=2,6.0 \%)$.

Considering $E$. coli $(\mathrm{n}=183)$ and anaerobic bacteria $(\mathrm{n}=$ 23) were the top two most frequently isolated pathogens in BSI among postpartum women; therefore, we analyzed the incidence rate of these two pathogens in patients suffering from PROM. As shown in Figure 2A, a total of 171 blood culture samples from postpartum patients who underwent cesarean section $(\mathrm{n}=220)$ were identified as E. coli, $55.5 \%$ of which were from those who underwent cesarean section complicated by PROM $(n=95)$. The number of postpartum patients who underwent vaginal delivery $(\mathrm{n}=26)$ who were infected with $E$. coli was $12,41.6 \%$ of whom underwent vaginal delivery combined with PROM $(n=5)$. These

\section{A Gram-negative bacteria}

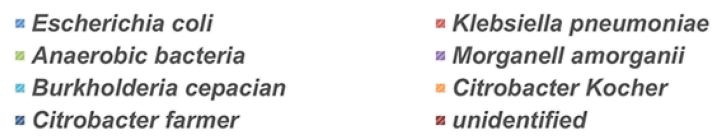

- Klebsiella pneumoniae * Morganell amorganii

- Citrobacter Kocher

s unidentified

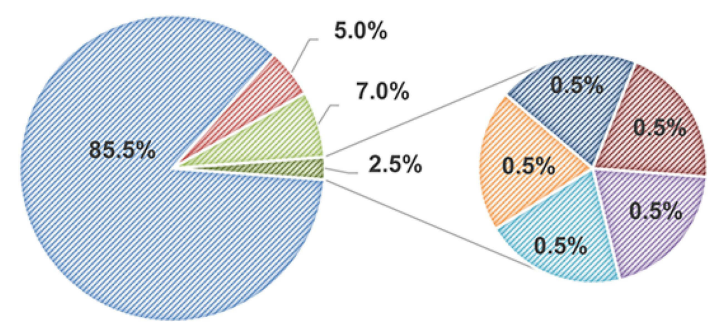

findings suggest that $E$. coli is the most common organism in BSI among postpartum patients, regardless of delivery mode, and the mechanisms through which $E$. coli causes BSI require further elucidation. Similarly, Figure 2B reveals no significant difference in the incidence of anaerobes in BSI between the cesarean section group and the vaginal delivery group in the context of PROM.

\section{Anaerobe Detection in BSI Among Postpartum Patients Increased Over the 6-Year Study Period}

The pathogenic spectrum can change in response to inappropriate antibiotic applications or the development of resistance. We observed the trends in the pathogenic spectrum from 2015 to 2020. As shown in Figure 3A, the detection rate of both gram-negative and gram-positive microorganisms in BSI peaked in 2020 overall. In addition, the detection rate of E. coli peaked in 2018 and again in 2020, as shown in Figure 3B. A slight downtrend in Klebsiella pneumoniae infections and an uptrend in Streptococcus agalactiae infections were observed for BSI over the 6-year study period. An obviously increasing tendency for the detection of anaerobes was observed between 2015 and 2020, suggesting the additional attention should be paid to BSI caused by anaerobes among postpartum patients.

\section{Higher Resistance Rate of E. coli Against Fourth-Generation Cephalosporin}

We then examined the sensitivities of the gram-negative $E$. coli in response to commonly used antibacterial drugs, as

\section{B Gram-positive bacteria}
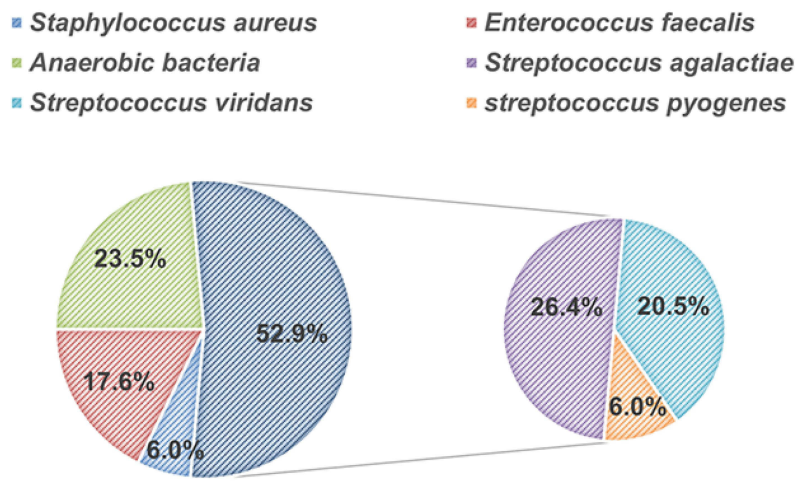

Figure I Pathogenic spectrum and distribution of BSls during the study period. The distribution and percentages of (A) gram-negative and (B) gram-positive pathogens isolated from blood culture samples in this study.

Abbreviation: BSI, bloodstream infection. 
A

\section{E. coli}

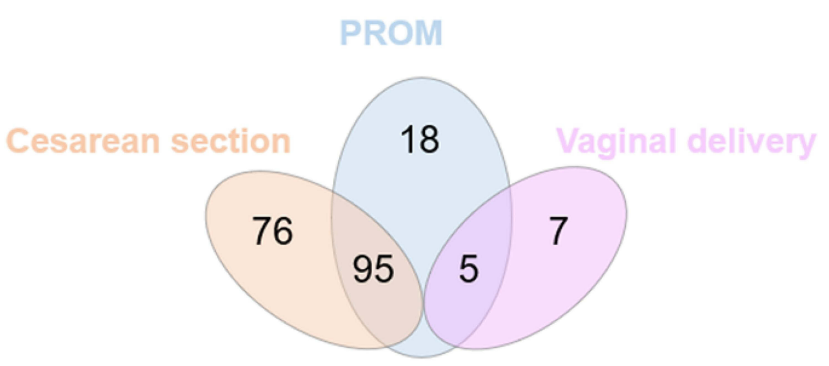

B

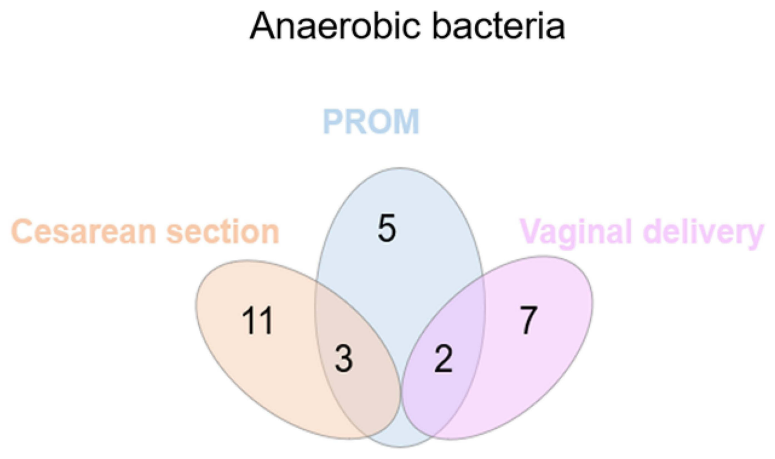

Figure 2 Analysis of the incidence rate of $E$. coli and anaerobes isolated in BSIs from patients according to cesarean or vaginal delivery due to PROM. Cases of patients infected with $E$. coli $(\mathbf{A})$ and anaerobic bacteria (B) under cesarean section (yellow) or vaginal delivery (purple) are shown. Cases of patients who underwent cesarean section or vaginal delivery due to PROM are shown.

Abbreviations: BSI, bloodstream infection; PROM, premature rupture of membranes; E. coli, Escherichia coli.
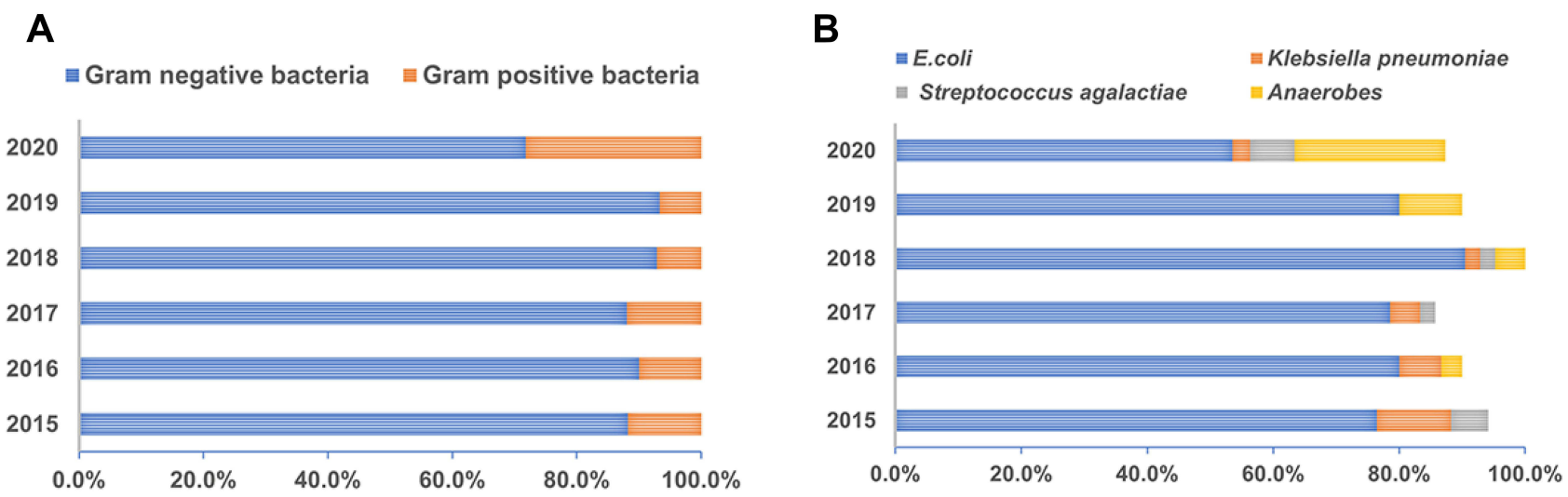

Figure 3 Changes in the pathogenic bacterial distribution in BSI from 2015 to 2020. (A) The overall distribution trend of pathogens isolated in BSIs from postpartum women between 2015 to 2020. (B) The detection of common pathogens in BSls in recent years.

Abbreviation: $\mathrm{BSI}$, bloodstream infection.

shown in Figure 4. Overall, E. coli exhibited general sensitivity to commonly used antibiotics, including $100 \%$ sensitivity in response to carbapenem antibiotics, over $50 \%$ sensitivities in response to aminoglycoside antibiotics and quinolone antibiotics. The drug resistance rate of $E$. coli against cefepime was approximately $20 \%$, whereas only an approximately $6 \%$ resistance rate was shown against ceftazidime.

We then specifically analyzed the sensitivity trends for $E$. coli in response to major, widely used antibiotics over the study period. As presented in Figure 5, the trend of resistance against ampicillin/subatan and cefazolin has been declining (Figure 5A and B), and a fluctuate trend of resistance against ceftazidime and cefepime was observed between 2015 and 2019 (Figure 5C and D). The proportion of critical patients and the increasing diversity of diseases may have contributed to the increasing trend in resistance observed for the year 2020. Notably, a higher resistance rate for $E$. coli against cefepime was observed during the 6-year period compared with the resistance to ceftazidime which warranted much attention.

\section{Discussion}

The present data showed that gram-negative microorganisms (especially E. coli) dominated BSIs during the past 6 years at our study centers. Additionally, an increasing trend in anaerobic bacteria detection in BSIs was observed among the postpartum patients in this study. Another point worthy of attention is the increasing resistance rate of $E$. coli against fourth-generation cephalosporins compared with resistance to third-generation cephalosporins during the study period. 


\section{Sensitivity of E.coli to common antibiotics}

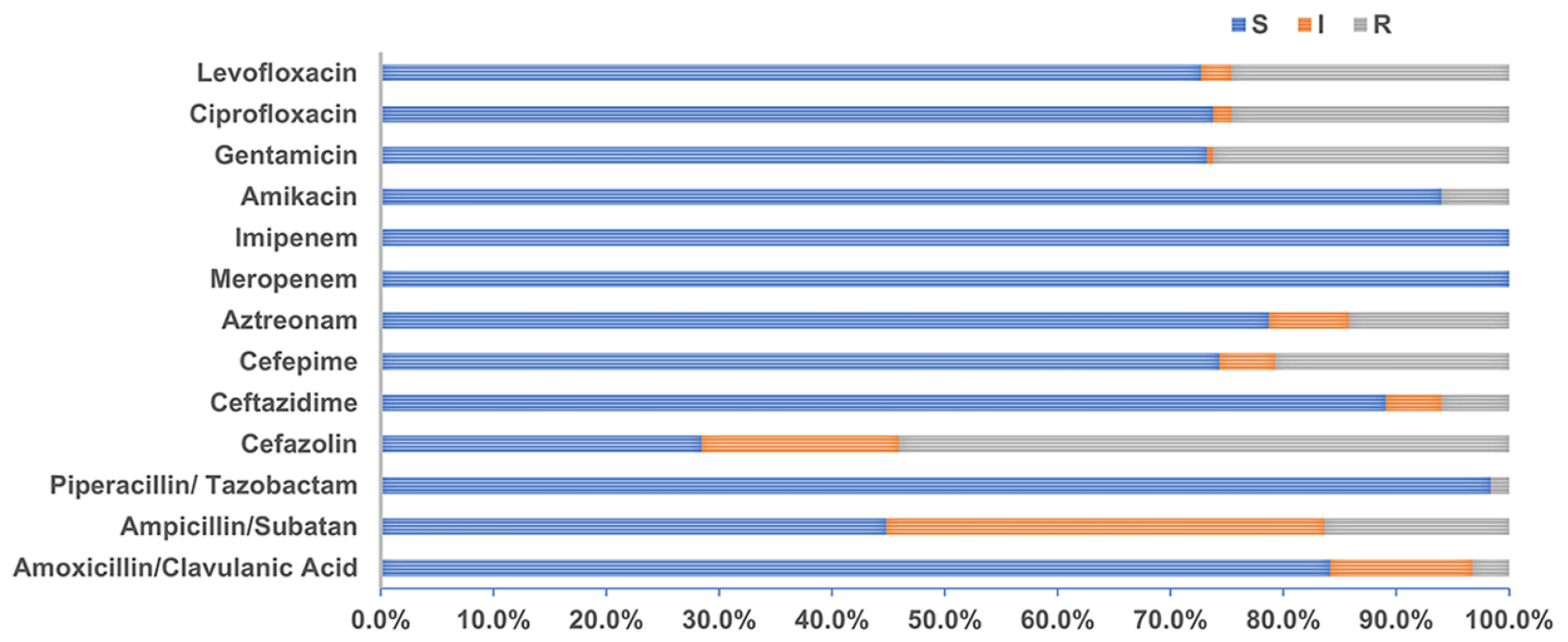

Figure 4 Sensitivity analysis of $E$. coli to common antibiotics.

Abbreviations: $\mathrm{S}$, sensitive; I, intermediate; R, resistant; E. coli, Escherichia coli.

A

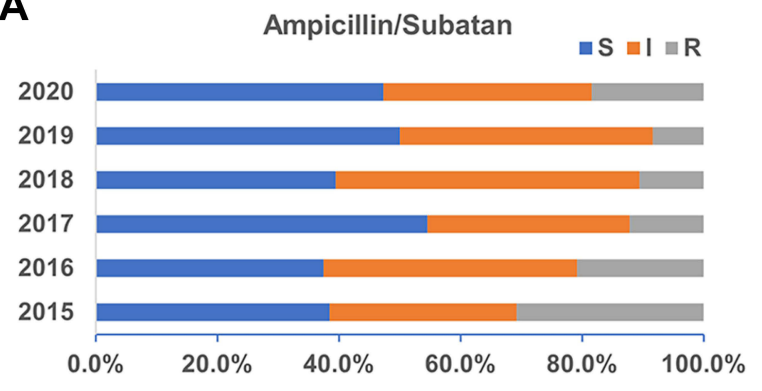

C

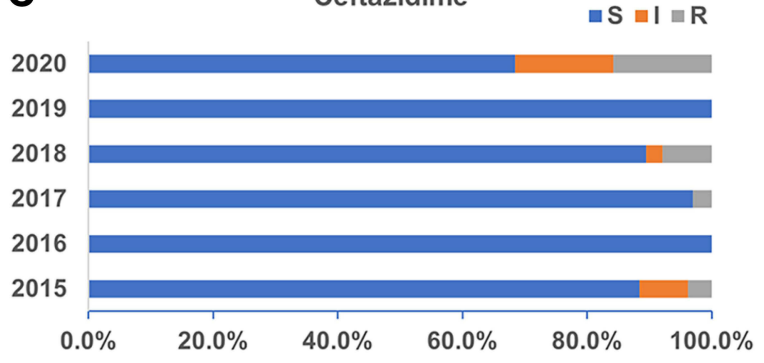

B

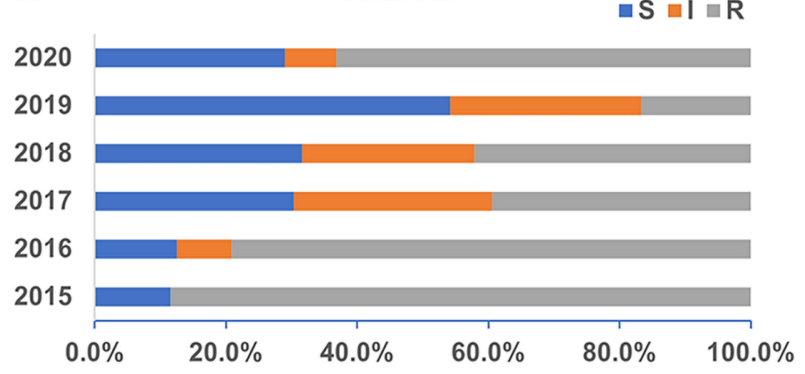

D

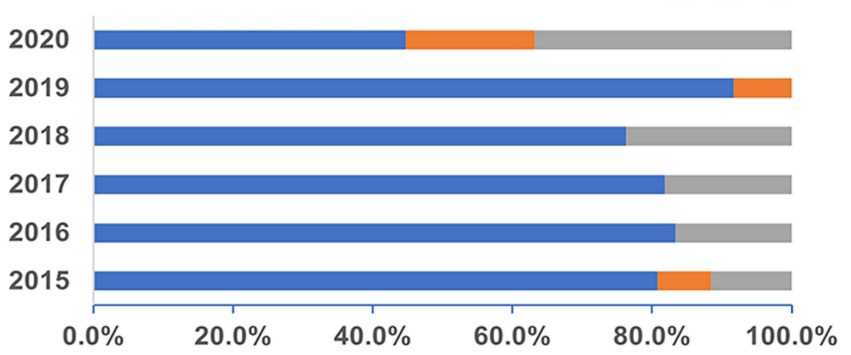

Figure 5 Sensitivity of E. coli to commonly used antibacterial drugs (\%). (A) Sensitivity of E. coli against Ampicillin/Subatan during the past 6 years. Sensitivity of E. coli against cephalosporins including (B) Cefazolin, (C) Ceftazidime, and (D) Cefepime.

Abbreviations: E. coli, Escherichia coli. S, sensitive; I, intermediate; R, resistant.

BSI represents an important cause of morbidity and mortality among postpartum patients and remains a substantial clinical problem. In this study, cases in which microorganisms were detected in at least two sets of positive blood cultures were defined as BSI, and the results suggested that $E$. coli has emerged as the predominant causative organism for BSIs, which is consistent with a recent study from China (BRICS report of 2016-2017), which identified $E$. coli as the major BSI-causing pathogen. ${ }^{14}$ However, reports from Malawi in Africa ${ }^{15}$ 
revealed Salmonella typhi and Streptococcus pneumoniae as the major BSI-causing pathogens. Another report from Rwanda demonstrated a varying pathogenic profile for BSIs, with Staphylococcus aureus and Klebsiella pneumoniae reported as the leading causative organisms. ${ }^{16}$ These discrepancies might be attributable to cultural, healthseeking behaviors, health care coverage, the period during which the studies were conducted, and other socioeconomic factors.

Although the most frequently detected pathogens remain consistent, their ranks may shift over time. In the present retrospective study, E. coli continued to rank the highest among detected bacteria, but the detection rate of anaerobic bacteria gradually increased to second place (23.9\%), according to the data in the year 2020 . Anaerobic bacteria remain an important cause of bloodstream infections, and the detection rate of anaerobes in blood cultures ranges from $1-17 \%$ across all bacteremic episodes, depending on patient age, condition, and geographic location. ${ }^{17,18}$ Recently, a report on the evaluation of the gut bacterial community composition in pregnant women concluded that Prevotella is significantly more abundant in the guts of pregnant women compare with those of non-pregnant women based on $16 \mathrm{~S}$ amplicon sequencing performed on stool samples. ${ }^{19}$ In this study, we found that $33.3 \%$ of anaerobic bacterial infections were identified as Prevotella bivia in BSIs, followed by Bacteroides fragilis (13.3\%) and Gardnerella vaginalis (13.3\%). Another study performed at a university hospital identified that Bacteroides fragilis (39.9\%) and Clostridium species (32.8\%) were the major anaerobic bacteria in BSIs. ${ }^{20}$ A different retrospective study revealed that $43.9 \%$ of anaerobic bacteria belong to the genus Bacteroides, $7 \%$ to the genus Fusobacterium, and $2.1 \%$ to the genus Prevotella. ${ }^{21}$ Currently, studies on the analysis of anaerobic bacteria in postpartum infections with BSI are scarce, additional attention should be paid to BSI caused by anaerobes among postpartum women, and more efforts should be spent identifying the potential sources of anaerobe infections and guiding clinicians in rational drug use according to population characteristics in the future.

The vaginal microbiome composition changes when women become pregnant, and in this study, two postpartum patients were confirmed with Gardnerella vaginalis infection, a well-organized colonizer of the female genital tract. Recent studies have shown that bacterial vaginosis (BV), which is dominated by Gardnerella vaginalis and can be caused by a number of anaerobic organisms, is linked with pelvic inflammatory disease, and BV-associated bacteria have been related to an increased risk of spontaneous abortions, preterm PROM, postpartum endometritis, and post-cesarean wound infections. ${ }^{22-25}$ Thus, effective diagnosis and treatment of BV may be significant for reducing the perinatal infection rate.

Another issue worthy of attention is the seriousness of extended antimicrobial resistance among gram-negative bacteria, and gram-negative organisms have been identified as a critical priority on the World Health Organization's global priority list of antibiotic-resistant bacteria. $^{26}$ The limited ability to treat such infections and the lack of new antimicrobial agent development can make BSIs associated with antibiotic-resistant bacteria challenging to treat. Better knowledge of antimicrobial resistance patterns will guide the actions of local and regional bodies as they work to counter antimicrobial resistance. Our data of antibiogram analysis indicated that $E$. coli isolates were generally susceptible to carbapenem antibiotics (100\%), and over $80 \%$ of $E$. coli isolates showed sensitivity to piperacillin/tazobactam (98.4\%), amikacin (94.0\%), ceftazidime (89.1\%), and amoxicillin/clavulanic acid (84.2\%). An increase in the number of critical patients and pregnancy complications may have contributed to the increased resistance rate of $E$. coli against third/fourthgeneration of cephalosporins in 2020 .

Recently, phenomenologically heteroresistance, a phenotype in which the treated bacteria contain subpopulations with lower susceptibility to the antibiotic than the dominant population, has been detected in clinical isolates ${ }^{27,28}$ and found to mediate antibiotic treatment failure. ${ }^{29}$ Here in our study, the resistance rate of $E$. coli to third-generation cephalosporins was $6.0 \%$, compared with the resistance rate of $20.8 \%$ against fourth-generation cephalosporins in the present study, pointing out the possibility of drug resistance heterogeneity contributes to this phenomenon. Increasing efforts are warranted to determine and counteract the development of antibiotic heteroresistance in E. coli isolated from blood culture samples of postpartum women.

Another finding in our study is that the onset of fever among participants typically occurred within two days after delivery, driving us to wonder whether effective prophylactic antibiotic use might affect the incidence of BSI in the context of the continued rise in the cesarean birth rate and the increased risk of surgical site infections after cesarean birth compared with vaginal birth. Indeed, ACOG has recently released a Practice Bulletin on the role 
of prophylactic antibiotics in labor and delivery. Timing is critically important because the goal is to achieve adequate tissue levels of antibiotics prior to pathogen exposure. ${ }^{30}$ Smaill $^{31}$ claimed that the incidence of wound infections, endometritis, and serious infectious complications would be reduced by $60 \%$ to $70 \%$ in response to the use of prophylactic antibiotics in women undergoing cesarean section. Previous study reported that the rate of infectious morbidity was similar among women who received prophylactic antibiotics within $30 \mathrm{~min}$ and 30-60 min before skin incision. ${ }^{32}$ Prophylactic antibiotics might be beneficial for women, but uncertainty regarding the consequences for the baby remains, which should be examined in future prospective research studies.

One limitation of the study was that it analyzed antibiograms of BSI caused by gram-negative bacteria from multicenter, which may not represent other populations, and the conclusions may therefore be difficult to generalize. Another limitation is the lack of molecular characterization for observed resistance. Due to the low-resource setting, resistance analyses for anaerobic bacteria that can cause significant puerperal sepsis were not performed. The results suggested that future research on BSI-causing pathogens should be expanded to cover other regions to obtain a more comprehensive understanding of the pathogenic profile and antimicrobial resistance patterns across regions, combined with the molecular characterization of the resistance mechanisms. Additionally, studies examining effective biomarkers capable of distinguishing infections caused by gram-negative or gram-positive organisms can facilitate the early initiation of appropriate antimicrobial therapy for better patient outcomes and are warranted in the future.

\section{Conclusion}

Our data showed that $E$. coli has dominated BSIs among postpartum patients over the past 6 years. Attention should be paid to the increasing trend in the detection of anaerobic bacteria in BSIs among postpartum women, and improved methods for identifying anaerobic bacteria and performing drug sensitivity testing should be implemented. The increasing resistance rate of E. coli against fourthgeneration cephalosporins in BSIs is also worthy of additional attention.

\section{Acknowledgments}

This work was sponsored by the Natural Science Foundation of Chongqing, China [grant no. cstc2020jcyjmsxmX0588].

\section{Disclosure}

The authors declare that they have no competing interests.

\section{References}

1. Van Dillen J, Zwart J, Schutte J, et al. Maternal sepsis: epidemiology, etiology and outcome. Curr Opin Infect Dis. 2010;23(3):249-254. doi:10.1097/QCO.0b013e328339257c

2. McCaw-Binns AM, Campbell LV, Spence SS. The evolving contribution of non-communicable diseases to maternal mortality in Jamaica, 1998-2015: a population-based study. BJOG. 2018;125 (10):1254-1261. doi:10.1111/1471-0528.15154

3. Hirshberg A, Srinivas SK. Epidemiology of maternal morbidity and mortality. Semin Perinatol. 2017;41(6):332-337. doi:10.1053/j. semperi.2017.07.007

4. Lapinsky SE. Obstetric infections. Crit Care Clin. 2013;29(3):509520. doi:10.1016/j.ccc.2013.03.006

5. Goto M, Al-Hasan M. Overall burden of bloodstream infection and nosocomial bloodstream infection in North America and Europe. Clin Microbiol Infect. 2013;19(6):501-509. doi:10.1111/1469-0691.12195

6. Yang ZY, Zhan SY, Wang B, et al. Fatality and secular trend of bloodstream infections during hospitalization in China. Beijing Da Xиe Bao Yi Xue Ban. 2010;42(3):304-307.

7. Bebell LM, Ngonzi J, Bazira J, et al. Antimicrobial-resistant infections among postpartum women at a Ugandan referral hospital. PLoS One. 2017;12(4):e0175456. doi:10.1371/journal.pone.0175456

8. Zhang X, Gu B, Mei Y, et al. Increasing resistance rate to carbapenem among blood culture isolates of Klebsiella pneumoniae, Acinetobacter baumannii and Pseudomonas aeruginosa in a university-affiliated hospital in China, 2004-2011. J Antibiotics. 2015;68 (2):115-120. doi:10.1038/ja.2014.119

9. Gopalakrishnan R, Sureshkumar D. Changing trends in antimicrobial susceptibility and hospital acquired infections over an 8 year period in a tertiary care hospital in relation to introduction of an infection control programme. J Assoc Physicians India. 2010;58(Suppl):25-31.

10. Jorak A, Keihanian F, Saeidinia A, et al. A cross sectional study on knowledge, attitude and practice of medical students toward antibiotic resistance and its prescription, Iran. Adv Environ Biol. 2014;8 (17):675-681.

11. Humphries RM, Abbott AN, Hindler JA. Understanding and addressing CLSI breakpoint revisions: a primer for clinical laboratories. J Clin Microbiol. 2019;57(6):e0203-19. doi:10.1128/JCM. 00203-19

12. Arendrup MC, Friberg N, Mares M, et al. How to interpret MICs of antifungal compounds according to the revised clinical breakpoints $\mathrm{v}$. 10.0 European committee on antimicrobial susceptibility testing (EUCAST). Clin Microbiol Infect. 2020:26(11):1464-1472. doi:10.1016/j.cmi.2020.06.007.

13. Yangco B. CDC definitions for nosocomial infections. Am J Infect Control. 1989;17(1):42-43. doi:10.1016/S0196-6553(89)80013-6

14. Chen YB, Ji JR, Ying CQ, et al. BRICS report of 2016-2017: the distribution and antimicrobial resistance profile of clinical isolates from blood culture in China. Chin J Clin Infect Dis. 2020;13(01):42-54.

15. Musicha P, Cornick JE, Bar-Zeev N, et al. Trends in antimicrobial resistance in bloodstream infection isolates at a large urban hospital in Malawi (1998-2016): a surveillance study. Lancet Infect Dis. 2017;17(10):1042-1052. doi:10.1016/S1473-3099(17)30394-8

16. Habyarimana T, Murenzi D, Musoni E, et al. Bacteriological profile and antimicrobial susceptibility patterns of bloodstream infection at Kigali University Teaching Hospital. Infect Drug Resist. 2021;23 (14):699-707. doi:10.2147/IDR.S299520

17. Diekema DJ, Beekmann SE, Chapin KC, et al. Epidemiology and outcome of nosocomial and community-onset bloodstream infection. $J$ Clin Microbiol. 2003;41(8):3655-3660. doi:10.1128/JCM.41.8. 3655-3660.2003 
18. Blairon L, De Gheldre Y, Delaere B, et al. A 62-month retrospective epidemiological survey of anaerobic bacteraemia in a university hospital. Clin Microbiol Infect. 2006;12(6):527-532. doi:10.1111/ j.1469-0691.2006.01407.x

19. Khan I, Yasir M, Farman M, et al. Evaluation of gut bacterial community composition and antimicrobial resistome in pregnant and nonpregnant women from Saudi population. Infect Drug Resist. 2019;12:1749-1761. doi:10.2147/IDR.S200213

20. Gajdács M, Terhes G, Urbán E. Az anaerob baktériumok által okozott véráramfertőzések gyakorisága 2005-2009 és 2013-2017 között egy egyetemi központban. Retrospektív összehasonlító vizsgálat [The incidence of bloodstream infections caused by anaerobic bacteria in a university hospital between 2005-2009 and 2013-2017. A retrospective, comparative study]. Orv Hetil. 2020;161(19):797-803. doi:10.1556/650.2020.31705

21. Cobo F, Borrego J, Gómez E, et al. Clinical findings and antimicrobial susceptibility of anaerobic bacteria isolated in bloodstream infections. Antibiotics (Basel). 2020;9(6):345. doi:10.3390/ antibiotics 9060345

22. Redelinghuys MJ, Ehlers MM, Dreyer AW, et al. Normal flora and bacterial vaginosis in pregnancy: an overview. Crit Rev Microbiol. 2016;42(3):352-363. doi:10.3109/1040841X.2014.954522

23. Brabant G. Vaginose bactérienne et prématurité spontanée [Bacterial vaginosis and spontaneous preterm birth]. J Gynecol Obstet Biol Reprod (Paris). 2016;45(10):1247-1260. doi:10.1016/j. jgyn.2016.09.014

24. Subtil D, Brabant G, Tilloy E, et al. Early clindamycin for bacterial vaginosis in pregnancy (PREMEVA): a multicentre, double-blind, randomised controlled trial. Lancet. 2018;392(10160):2171-2179. doi:10.1016/S0140-6736(18)31617-9
25. Soper DE. Bacterial vaginosis and surgical site infections. Am J Obstet Gynecol. 2020;222(3):219-223. doi:10.1016/j. ajog.2019.09.002

26. Shrivastava SR, Shrivastava PS, Ramasamy J. Responding to the challenge of antibiotic resistance: World Health Organization. J Res Med Sci. 2018;23:21. doi:10.4103/1735-1995.228593

27. Nicoloff H, Hjort K, Levin BR, et al. The high prevalence of antibiotic heteroresistance in pathogenic bacteria is mainly caused by gene amplification. Nat Microbiol. 2019;4(3):504-514. doi:10.1038/ s41564-018-0342-0

28. Sun JD, Huang SF, Yang SS, et al. Impact of carbapenem heteroresistance among clinical isolates of invasive Escherichia coli in Chongqing, southwestern China. Clin Microbiol Infect. 2015;21 (5):469.e1-10. doi:10.1016/j.cmi.2014.12.013

29. Band VI, Crispell EK, Napier BA, et al. Antibiotic failure mediated by a resistant subpopulation in Enterobacter cloacae. Nat Microbiol. 2016;1(6):16053. doi:10.1038/nmicrobiol.2016.53

30. ACOG Practice Bulletin No. 199: use of prophylactic antibiotics in labor and delivery: correction. Obstet Gynecol. 2018;132(3):e103e119. Erratum in Obstet Gynecol. 2019;134(4):883-884. doi:10.1097/AOG.0000000000003499

31. Smaill FM, Grivell RM. Antibiotic prophylaxis versus no prophylaxis for preventing infection after cesarean section. Cochrane Database Syst Rev. 2014;2014(10):CD007482.

32. Rubin H, Rom E, Wattad M, et al. Effectiveness of antimicrobial prophylaxis at 30 versus $60 \mathrm{~min}$ before cesarean delivery. Sci Rep. 2021;11(1):8401. doi:10.1038/s41598-021-87846-Z
Infection and Drug Resistance

\section{Publish your work in this journal}

Infection and Drug Resistance is an international, peer-reviewed openaccess journal that focuses on the optimal treatment of infection (bacterial, fungal and viral) and the development and institution of preventive strategies to minimize the development and spread of resistance. The journal is specifically concerned with the epidemiology of antibiotic resistance and the mechanisms of resistance development and diffusion in both hospitals and the community. The manuscript management system is completely online and includes a very quick and fair peerreview system, which is all easy to use. Visit http://www.dovepress.com/ testimonials.php to read real quotes from published authors. 